\title{
Buchrezensionen
}

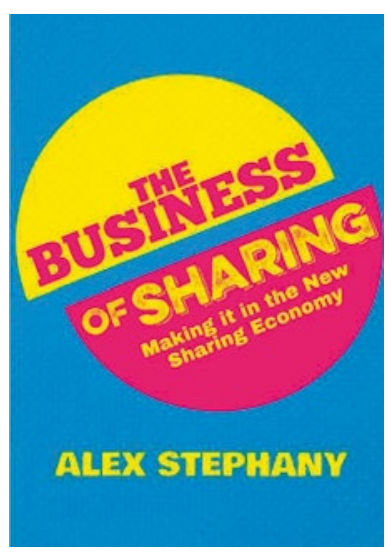

Alex Stephany

\section{The Business of Sharing}

Making it in the New Sharing Economy

1. Auflage, Palgrave Macmillan, London

2015, 210 Seiten, 25,62 Euro

ISBN 978-1-137-37617-6

\section{Kernthesen}

- Der Begriff des Eigentums verändert sich rapide.

- Wir bewegen uns hin auf ein sozioökonomisches System, welches auf die gemeinsame Nutzung unserer Zeit, Talente und Ressourcen aufbaut.

- Die „Sharing Economy“ stellt die bisherigen Regeln des wirtschaftlichen Handelns auf den Kopf - und birgt eine Vielzahl an Chancen und Risiken.

\section{Nutzen für die Praxis}

Das Buch liefert eine umfassende Darstellung der neuesten Entwicklungen im Bereich der "Sharing Economy“. Alex Stephany beschreibt sowohl seine eigenen Erfahrungen als CEO von JustPark als auch die einer Vielzahl seiner bekannten Gründerkollegen wie Robin Chase von Zipcar oder Airbnb's Nathan Blecharczyk und präsentiert leicht verständlich und unterhaltsam die wichtigsten Erkenntnisse der Brancheninsider.

\section{Abstract}

Schon heute ermöglicht die "Sharing Economy“ oder der "kollaborative Konsum“ es, Privatpersonen mehr als 15 Milliarden US-Dollar pro Jahr durch die Vermietung oder den Verkauf von Teilen ihres nicht genutzten privaten Eigentums an Autos, Häusern, Geld und Zeit zu erwirtschaften. Und dies ist erst der Anfang: Laut PwC wird die „Sharing Economy“ bis 2025 auf eine Größe von 335 Milliarden US-Dollar anwachsen.

Die Idee dahinter ist simpel: Warum sollte man sich eine Heckenschere kaufen, die man nur zweimal pro Jahr benutzt? Stattdessen könnte man sie sich doch einfach bei jemandem ausleihen. Oder die heimische Garage. Warum sollte sie tagsüber ungenutzt bleiben, wenn es genügend Parkplatzsuchende im Umkreis gibt?

Genau solche Kapazitäten machen sich Unternehmen wie RelayRides oder thredUP zunutze. Sie mobilisieren Millionen Kleinstunternehmer und beginnen, riesige Märkte der „Old Economy“ zu zerrütten. Die weltgrößten Unternehmen müssen sich in Acht nehmen - Investoren des Silicon Valley pumpen hohe Summen in die Sharing Economy. Können Konzernriesen lernen, in solch einem Umfeld nicht nur zu überleben, sondern in der Welt des Peer-to-Peer-Handels zu florieren?

Das Buch ist eine ideale Lektüre für alle diejenigen, die einen authentischen Einblick in den rapide wachsenden globalen Trend der "Sharing Economy" gewinnen und von den Erfahrungen der Top-Brancheninsider lernen möchten.

Selina Wilke 